\title{
МЕТОДИКА НАБЛЮДЕНИЙ И РЕЗУЛЬТАТЫ АЭРОМАГНИТНОЙ СЪЁМКИ ПО ВОСТОЧНОЙ ЧАСТИ СУЭЦКОГО ЗАЛИВА (ЕГИПЕТ)
}

\author{
А. Таршан ${ }^{1,2}$ \\ ${ }^{1}$ Санкт-Петербургский государственный университет, \\ ${ }^{2}$ Управление по ядерным материалам, Каир, Египет
}

Поступила в редакцию 22 мая 2019 г.

\begin{abstract}
Аннотация: по сравнению с другими геофизическими методами, аэромагнитный метод значительно чаще применяется при поиске и разведке месторождений полезных ископаемых и в больиинстве случаев он дает лучшие результаты. Данные аэромагнитных измерений в этом исследовании были получены вдоль основных профиле (находящихся на расстоянии 1000 м друг от друга) и вдоль контрольных профилей, находящихся на расстоянии 10000 м друг от друга и перпендикулярных основным марирутам. Номинальное летное превышение (относительная высота залета) составляло около 100 м над земной поверхностью. Представлены общие сведения о калибровке данных. По отношению к самим данным использовалась определенная последовательность шагов их обработки. На основании карты индукиии магнитного поля составлена карта приведения к полюсу. Результаты показали, что в исследуемом районе есть две основные аномальные зоны. Первая зона расположена в восточной части района, распространяющейся на юго-западную часть, которая характеризуется сильной амплитудой магнитного склонения. Вторая зона находится в западной и юго-восточной частях района, и характеризуется слабой амплитудой магнитного склонения.
\end{abstract}

Ключевые слова: обработка данных аэромагнитных измерений, приведение к полюсу, напряженность магнитного поля.

\section{OBSERVATION METHODOLOGY AND RESULTS OF AEROMAGNETIC SURVEY IN THE EASTERN PART OF THE GULF OF SUEZ (EGYPT)}

\author{
A. Tarshan ${ }^{1,2}$ \\ ${ }^{1}$ Saint Petersburg State University \\ Nuclear Materials Authority, Cairo, Egypt
}

Received 22 May 2019

\begin{abstract}
: the airborne magnetic method has more applications in prospecting and exploration for mineral deposits comparing to other airborne geophysical methods, and mainly leads to better results. The airborne magnetic data was acquired along primary lines spaced at $1000 \mathrm{~m}$ and along control lines spaced at $10000 \mathrm{~m}$, (normal to the primary lines. Nominal flying elevation was about $100 \mathrm{~m}$ above ground surface (terrain clearance). An overview of the calibration and processing steps of the airborne magnetic data is presented and applied on the data. The Reduction to pole (RTP) map is created from total magnetic intensity map. Results showed that there are two major zones within the study area. The first zone is located in the Eastern side of the area

extended to southwestern part of the area which is characterized by high magnetic amplitude. The second zone encountered in the western and southeastern side of the area and characterized by low magnetic amplitude.
\end{abstract}

Key words: processing of Airborne magnetic data, Reduction to the pole, Total magnetic intensity. 


\section{Введение}

Аэромагнитная съемка - один из лучших методов определения глубины залегания слоя с намагниченными источниками в геологической среде [1]. Тремя основными стадиями данного метода (также как и других геофизических методов) являются: получение, обработка и интерпретация данных [2]. Стадия получения данных включает различные методы, которые обычно классифицируются по двум категориям: данные с вертолетов и самолетов. Целью обработки данных магниторазведки является выделение компонентов магнитного поля, возникающего вследствие влияния корового материала, путем применения этапов обработки. Стадия интерпретации данных включает следующие методы: собственно интерпретацию, моделирование и определение параметров земной поверхности (включая глубину источников магнитных аномалий).

План аэросъемки обычно выполняется посредством осуществления полетов вдоль серии эквидистантных профилей (линий) залетов. В целях составления карт по общим рекогносцировочным данным направление профилей обычно ориентировано с севера на юг или с востока на запад, в зависимости от преобладающего простирания известных геологических структур [3]. Качество результатов аэромагнитной съемки зависит от точности обработки данных аэромагнитных измерений. Обработка данных аэромагнитных измерений разделяется на две этапа: первый - это этап предварительной обработки (калибровки), используемый в полевых условиях, второй же этап выполняется после окончания съемки.

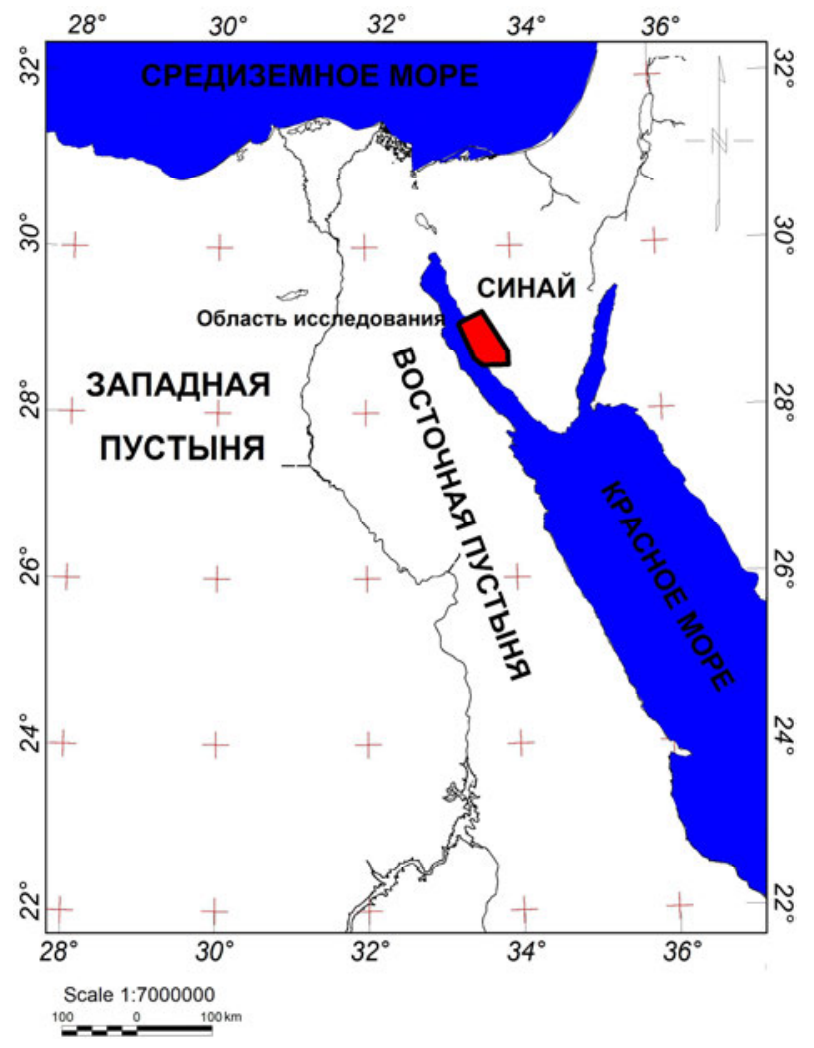

Puc. 1. Схема расположения изучаемого района.

\section{Данные}

Служба аэрогеофизических работ при Управлении по исследованиям ядерных материалов (УИЯМ) Египта провела аэромагнитную съемку высокого разрешения для района, охватывающего 2745 км² $^{2}$ рис. 1) в восточной части Суэцкого залива (Египет). Съемка выполнялась с двухмоторного самолета с газотурбинными двигателями King Air Beechcraft C90B, оборудованного развитой системой аэрогеофизической съемки, использующей большое количество датчиков.

Данные были получены при полете вдоль основных линий съёмки (находящихся на расстоянии 1000 м друг от друга), а также вдоль контрольных линий, находящихся на расстоянии 10000 м друг от друга и перпендикулярных основным линиям. Номинальное летное превышение (высота над местностью) составляло около 100 м над земной поверхностью. Ориентирование съемки составляло $125^{\circ}-305^{\circ}$ по азимуту для основных линий и $35^{\circ}-215^{\circ}$ по азимуту для контрольных линий. Магниторазведочные данные фиксировались с частотой дискретизации 0,1 сек. При такой частоте самолет пролетал вдоль профиля съёмки пролета примерно 70 м за секунду.

\section{Калибровка данных}

\section{Испьтание по показателю качества}

Данный калибровочный облет выполняется на большой высоте (10 тыс. фут или 3048 м) вдоль сторон прямоугольника, представляющего основные направления полета при съемке. В испытания вошла серия поперечных наклонов, продольных наклонов и рысканий вдоль каждой стороны прямоугольника под углами $\pm 10^{\circ}, \pm 5^{\circ}$ и $\pm 5^{\circ}$, соответственно.

Эти маневрирования (тангажи, крены, и рыскания) вдоль каждой из четырех сторон были ясно отделены в пространстве и времени для того, чтобы визуально наблюдать и рассчитывать влияние каждого маневра до и после динамической компенсации. Как необработанные (нескорректированные) маневры, так и динамически компенсированные (скорректированные) маневры были нанесены на график для визуального определения вариаций максимумаминимума маневра на результаты измерений магнитного поля. Расчетное значение показателя качества измерений составило 0,25 (nT).

\section{Испьтание по курсу}

Испытание проводилось в области низкого магнитного градиента и над визуально идентифицируемым топографическим объектом (что отслеживалось с помощью бортовой видеокамеры). Рассчитанные значения курса для основных линий и узловых контрольных линий составили \pm 10 и \pm 6 (nT), соответственно, что является вполне приемлемым для наблюдаемых полей вдоль четырех направлений съемки.

Испытание на «параллакс / запаздывание»

Это тестирование выполнялось посредством пролета над топографически опознаваемым и сильно 
проявляющимся магнитоактивным объектом. Полет с перекрытием выполнялся по направленному вперед и обратному курсу с использованием видеокамеры и глобальной навигационной спутниковой системы (GPS). Точное положение аномалии определялось по зафиксированным в цифровом формате магнитометрическим данным относительно референтных и синхронизированных по времени данных с видеоэкрана в каждой точке маршрута. Расчетная результативная ошибка «параллакс / запаздывание» составила 0,6 сек.

\section{Обработка данных}

Величина полного магнитного поля Земли, определенная в конкретной точке пространства, включает следующие компоненты: 1. Постоянное магнитное поле Земли. 2. Поле, обусловленное влиянием магнитоактивного корового материала. 3. Периодические и апериодические вариации магнитного поля Земли. 4. Полевые погрешности во время получения данных. 5. Магнитные аномалии искусственных сооружений.

Целью обработки данных магниторазведки является выделение компонентов магнитного поля, возникающего вследствие влияния корового материала, в то время как другие компоненты устраняются в процессе последующих шагов обработки.

Во-первых, применяется поправка на суточное изменение. Это ежесуточное изменение напряженности магнитных полей, которое происходит вследствие влияния солнечного ветра. Регистрируются непрерывной записью величины магнитного поля при стоянии в одной точке. Установлено, что интенсивность изменяется на заметную величину за короткие промежутки времени. Эти вариации имеют более или менее регулярный ежедневный цикл, который примерно одинаков в одно и то же солнечное время в разных точках [4]. Магнитометр опорной станции использовался для того чтобы извлекать суточные колебания путем измерения вариации с использованием магнитометра во время съемки, данные которые были получены от фиксированного магнитометра (магнитометра опорной станции) вносились в корректировку данных прямым вычитанием. Вовторых, выполняется коррекция курса. Роль курсовой коррекции заключается в коррекции данных при систематическом смещении внутри самих данных. Такое систематическое смещение является функцией направления съемочного маршрута. В-третьих, выполняется поправка на запаздывание. Поправка на запаздывание должна использоваться в связи с расстоянием между датчиком и навигационной точкой вдоль линии самолета. Запаздывание - это время, которое требуется датчику для его перемещения в точку, принимаемую за положение самолета в данный момент времени. Поправка на запаздывание вычитает запаздывание (в опорных точках) от доверительного начала канала передачи данных в базе данных. Расчетная результативная погрешность «параллакс / запаздывание» составила 0,6 сек.

В-четвертых, значение международного эталона геомагнитного поля (IGRF) вычитается из наблюдае- мых данных. Это стандартное математическое описание крупномасштабной структуры постоянного магнитного поля Земли и его векового изменения [5]. Современный вариант модели IGRF был опубликован в 2015 г. [6] и действителен с 1900 г. по 2020 г. Пятый и шестой этапы: данные уравнивания и микроуравнивания. Причина необходимости уравнивания заключается в том, что при различных видах геофизической съемки данные получаются при постоянно меняющихся условиях, которые вызывают погрешности. Наиболее очевидный результат таких погрешностей это смещение видимого уровня в соседних параллельных съемочных маршрутах. Микроуравнивание означает устранение любых видимых остаточных погрешностей в аэрогеофизических данных после применения стандартного графа обработки, и после применения стадий уравнивания. Очень важно провести этап микроуравнивания для исключения влияния негеологических шумов, которые появляются в массиве данных.

Следующая методика уравнивания использовалась для получения приемлемо уравновешенного массива данных: 1. Статистическое уравнивание для контрольных профилей. 2. Микроуравнивание для контрольных профилей. 3. Сплайн уравнивание по линии профиля с использованием уравненных контрольных профилей. 4. Микроуравнивание линий профиля. 5. Сплайн уравнивание контрольных профилей скорректированных по IGRF с использованием линий профиля.

Результативные магниторазведочные данные наносились при очень точном расстоянии между линиями координатной сетки (250 м) с использованием минимального интерполирования линии отклонения от курса. Без такого интерполирования на результативных изображениях появятся «белые пятна» между линиями залета. Такие «белые пятна» усложняют интерпретацию данных [7].

Карта аномального магнитного поля приведения к полюсу (RTP) (рис. 2), была составлена по первичной полностью откорректированной карте аномального поля (рис. 3). Метод приведения к полюсу обычно применяется в частотной области, которая имеет некоторые недостатки, такие как индукция шума, необходимость использования фиксированного наклона и склонения по всей области съемки, а также неизвестная остаточная намагниченность, что во многих случаях ограничивает ее применимость. Вследствие наклонения магнитного поля Земли большая часть магнитных аномалий показывает как положительные, так и отрицательные экстремумы. Такие минимумы и максимумы, как правило, уравновешиваются из центра возмущающего объекта, расположенного вдоль магнитного меридиана. Только в том случае, когда наклонение составляет 90, магнитная аномалия находится непосредственно над центром тела источника магнитного излучения. Метод приведения к полюсу используется для исключения этого явления, поэтому данные появляются, как если бы это наблюдалось над полюсом, где магнитное поле является 


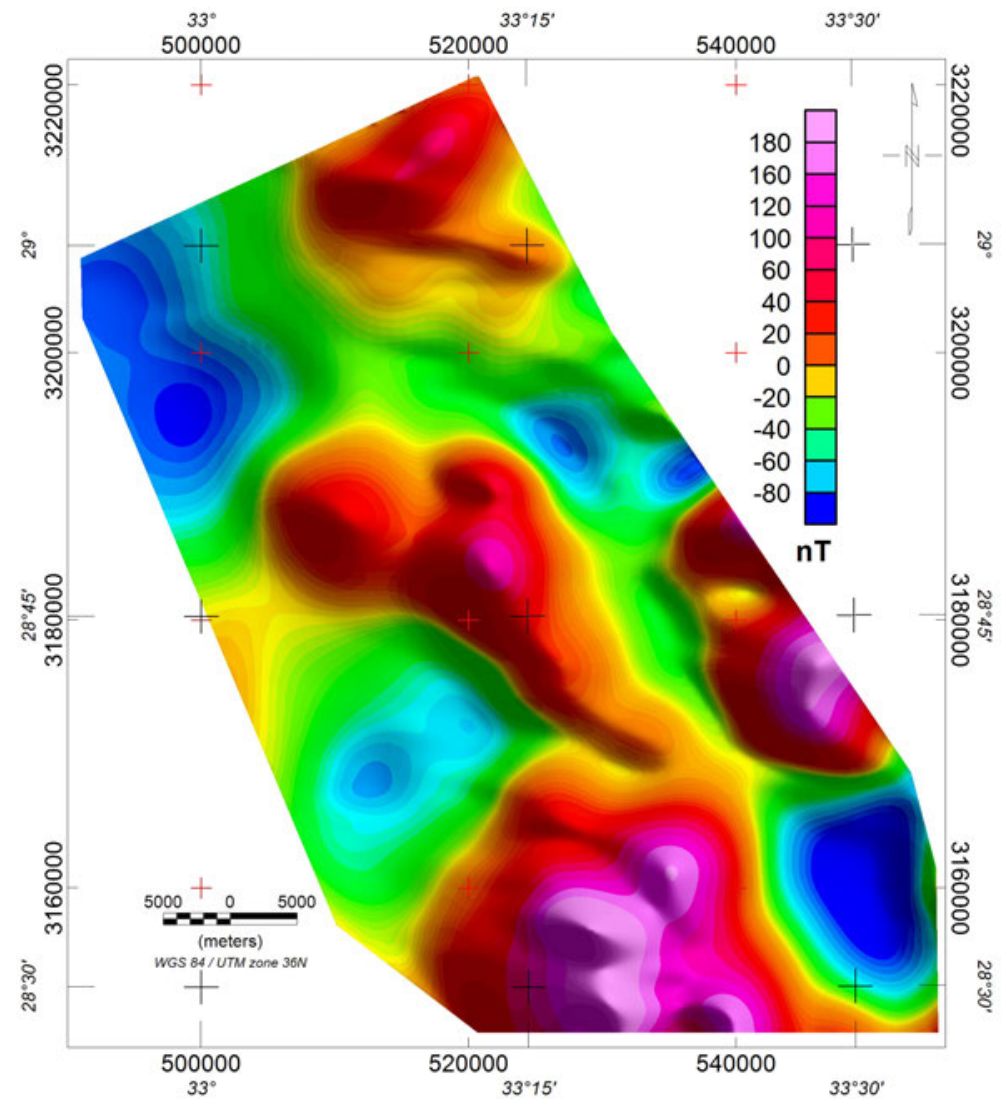

Puc. 2. Карта аномального магнитного поля приведённого к полюсу.

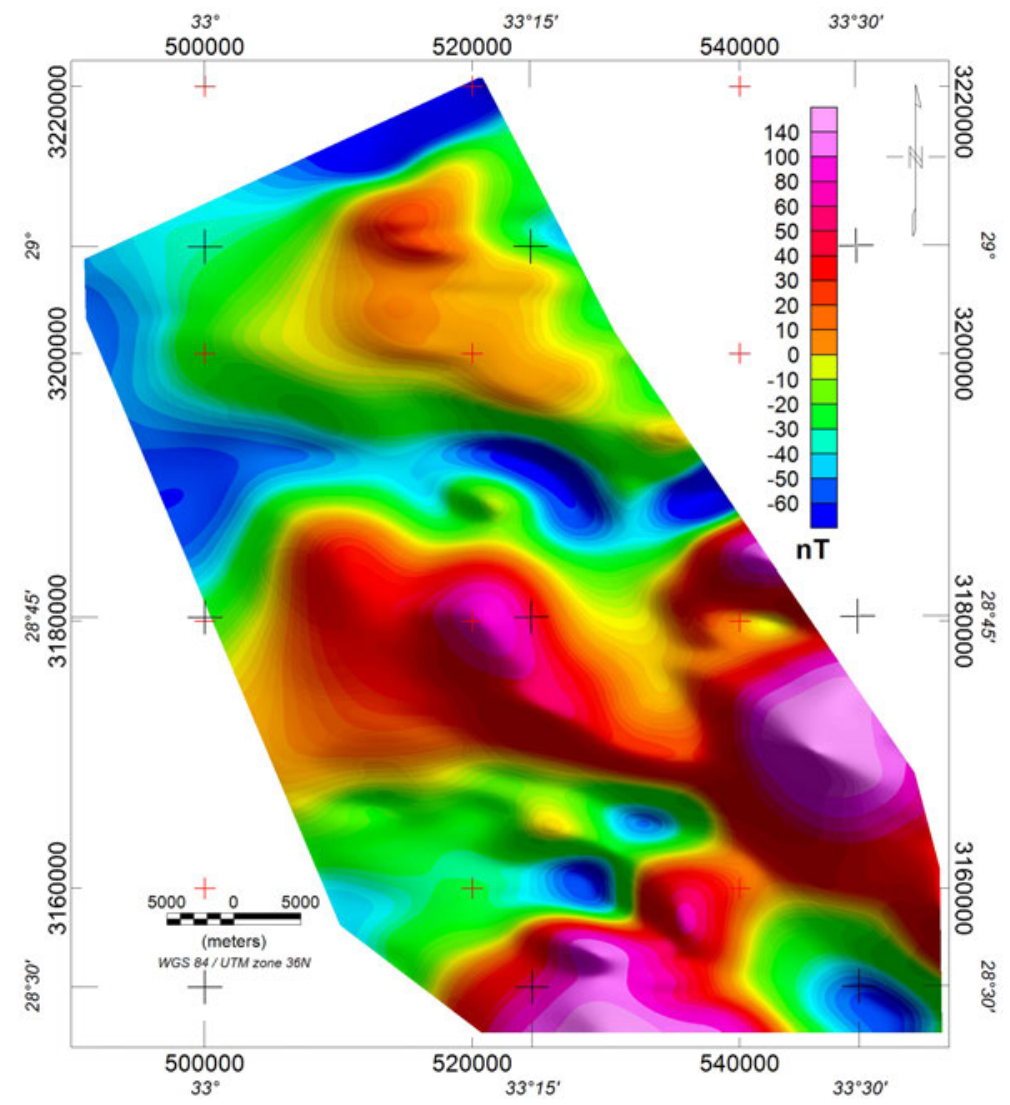

Puc. 3. Карта откорректированного наблюдённого аномального магнитного поля. 
вертикальным. Затем имеет место магнитный максимум непосредственно над намагниченными объектами, особенно в случае отсутствия остаточной намагниченности [8]. В противоположном случае, когда наклонение составляет $0^{\circ}$, минимум расположен непосредственно над магнитным объектом.

\section{Заключение}

Рассмотрены методология и логическая схема исследования по обработке данных аэромагнитных измерений. Во-первых, из набора данных устранялись суточные вариации, и из данных вычиталось региональное поле Земли. Исследование влияния изменения высоты на зарегистрированное магнитное поле показало, что различия между данными зарегистрированного магнитного поля вдоль линий залета и секущих маршрутов в изучаемых районах были обусловлены главным образом суточными вариациями. Изменения высот не выявили какой-либо корреляции с интенсивностью полного магнитного поля Земли. Это подразумевает, что различия между зарегистрированным магнитным полем вдоль линий залёта и секущими маршрутами не были обусловлены изменениями высоты. Для уравнивания аэромагнитных данных использовались различные методики; данные уравнивались с помощью методики секущего маршрута. В методике уравнивания использовались: статистическое уравнивание для контрольных профилей, микроуравнивание для контрольных профилей, сплайн уравнивание по линии профиля с использованием микроуравниванных контрольных профилей, микроуравнивание линий профиля и сплайн уравнивание контрольных профилей скорректированных по IGRF c использованием линий профиля.

В результате уравнивания контрольных профилей в наборе данных однозначно исключены ошибки направления. Приведение к полюсу (RTP) проводи- лось по полностью откорректированной карте индукции магнитного поля. Итоговые данные магнитной карты иллюстрирует местоположение положительных и отрицательных магнитных аномалий.

\section{Благодарности}

Автор благодарит Сергея Шиманского, доцента Кафедры геофизики Института наук о Земле при Санкт-Петербургском университете, за его помощь в решении различных проблем в ходе проведения работ.

\section{ЛИТЕРАТУРА}

1. Shimanskiy, $S$. $V$. Basement configuration depth methods of airborne magnetic data in the eastern Gulf of Suez, Egypt / S. V. Shimanskiy, A. Tarshan // News of Ural state Mining University Journal. - 2019. - V. 53 - P. 7- 17.

2. Rouhani, A. K. Processing and interpretation of airborne magnetic data for prospecting chromite deposits in the Sabzevar area / A. K. Rouhani, M. Beiki // Journal of the Earth and Space Physics. - 2009. - V. 35(3) - P.25-38.

3. Horsfall, K. R. Airborne magnetic and gamma-ray data acquisition / K. R. Horsfall // Journal of Australian Geology \& Geophysics. - 1997 - V. 17(2) - P. 23-30.

4. Nettleton, L. L. Gravity and magnetic in oil prospecting / L. L. Nettleton. - New YorkMcGraw-Hill Book Co., Inc, 1976. $464 \mathrm{p}$.

5. Dobrin, M. B. Introduction to geophysical prospecting / M. B. Dobrin. - McGraw-Hill Book Co., New York, USA, 1976. $630 \mathrm{p}$.

6. NOAA/NGDC candidate models for the $12^{\text {th }}$ Generation International Geomagnetic Reference Field / P. Alken [et al.] // Earth Planets Space. - 2015. - V. 67(68) - P. 1-9.

7. Duval, J. S. Composite colour images of aerial gamma-ray spectrometric data / J. S. Duval // Geophysics. - 1983. - V. 48 (16) - P. 722-735.

8. Kearey, $P$. An introduction to geophysical exploration/ $\mathrm{P}$. Kearey, B. Michael // Blackwell Scientific Publication, Second Edition, London, Great Britain. -1994. - P. 245.

Saint Petersburg State University Nuclear Materials Authority, Cairo, Egypt

Ahmed Tarshan, postgraduate student of the Department of Geophysics

E-mail: ahmed_ramadan_geo@hotmail.com

Tel.: +7(812) $3 \overline{6} 36196$ 